\title{
Application of Legendre Polynomial in Annual Electricity Consumption
}

\author{
Hui-jie JIANG* \\ College of Information and Technology, \\ Jilin Agricultural University \\ Changchun, China 130118 \\ Jianghuijie6058@126.com
}

\author{
Hong-qin ZHANG \\ College of Information and Technology, \\ Jilin Agricultural University \\ Changchun, China 130118 \\ zhanghq_@126.com
}

\begin{abstract}
National Statistical Bureau of China, and giving the least-squares fitting of Legendre polynomial, the data of electricity consumption in per capita from 1983 to 2013 is analyzed by GM $(1,1)$ method and Legendre polynomial least squares method respectively. The results showed that Legendre polynomial least squares fitting method is excellent, per capita energy consumption from 2014 to 2018 is predicted by this method.
\end{abstract}

\section{Keywords-GM (1,1); least squares; Legendre polynomial}

In the scientific research and production practice, the data obtained from observation, prediction and calculation should be analyzed and processed, and then the conclusions concerning the problems can be found out. The polynomial least square method is an effective method, because the accuracy and reliability of the least square method relies on the condition number of the coefficient matrix of its normal equation. Legendre polynomial is adopted as basis function, so as to make the small condition number of coefficient matrix of the normal equation of the least square method, and then the accuracy and reliability of the least square method are improved obviously. As China's increasingly affluent urban and rural living and modern, per capita electricity consumption increasing every year, energy conservation has become important part of China's energy conservation. Therefore, that the electricity consumption per capita is predicted accurately is very important for socioeconomic development.

\section{BASIC THEORY}

\section{A. $\quad G M(1,1)$ Prediction Model [1] [2]}

Let non-negative sequence $X^{(0)}$ of the original data sequence as follow

$$
X^{(0)}=\left\{X^{(0)}(1), X^{(0)}(2), \cdots, X^{(0)}(n)\right\}
$$

Where

$$
X^{(0)}(k) \geq 0 \quad k=1,2, \cdots, n
$$

The sequence $X^{(1)}$ obtained is as follow

$$
X^{(1)}=\left\{X^{(1)}(1), X^{(1)}(2), \cdots, X^{(1)}(n)\right\}
$$

Where

$$
X^{(1)}(k)=\sum_{i=1}^{k} X^{(0)}(i) \quad k=1,2, \cdots, n
$$

$Z^{(1)}$ Denotes a sequence close to the mean

$$
Z^{(1)}=\left\{Z^{(1)}(2), Z^{(1)}(3), \cdots, Z^{(1)}(n)\right\}
$$

Where

$$
\begin{gathered}
Z^{(1)}(k)=0.5 X^{(1)}(k)+0.5 X^{(1)}(k-1) \\
k=2,3, \cdots, n
\end{gathered}
$$

Named $x^{(0)}(k)+a z^{(1)}(k)=b$ the GM $(1,1)$ model, Where $a, b$ is solution of the model parameters?

$$
Y_{n}=\left(x^{(0)}(2), x^{(0)}(3), \ldots, x^{(0)}(n)\right)^{T}
$$

$$
B=\left[\begin{array}{cc}
-\frac{1}{2} Z^{(1)}(2) & 1 \\
-\frac{1}{2} Z^{(1)}(3) & 1 \\
: & : \\
-\frac{1}{2} Z^{(1)}(n) & 1
\end{array}\right]
$$

Algorithm for parameters with a minimum two columns $\hat{a}$ 


$$
\hat{a}=\left(\begin{array}{l}
a \\
b
\end{array}\right)=\left(B^{T} B\right)^{-1} B^{T} Y_{n}
$$

$N \alpha \mu \varepsilon \delta \delta \frac{d x^{(1)}}{d t}+a x^{(1)}=b$

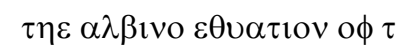

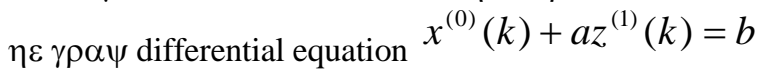

Albino equation or that the time response function

$$
\hat{x}^{(1)}(t)=\left(x^{(1)}(0)-\frac{b}{a}\right) e^{-a t}+\frac{b}{a}
$$

$\mathrm{GM}(1,1)$ gray differential equation

$$
x^{(0)}(k)+a z^{(1)}(k)=b
$$

For the time response

$$
\begin{gathered}
\hat{x}^{(1)}(k+1)=\left(x^{(1)}(0)-\frac{b}{a}\right) e^{-a k}+\frac{b}{a} \\
k=1,2, \cdots, n
\end{gathered}
$$

Take

$$
x^{(1)}(0)=x^{(0)}(1)
$$

$$
\hat{x}^{(1)}(k+1)=\left(x^{(0)}(1)-\frac{b}{a}\right) e^{-a k}+\frac{b}{a} \quad k=1,2, \cdots, n
$$

Restore value

$\hat{x}^{(0)}(k+1)=\hat{x}^{(1)}(k+1)-\hat{x}^{(1)}(k) \quad k=1,2, \cdots, n$

B. The linear least square of Legendre polynomial

The expression of the Legendre polynomial is that:

$$
\begin{aligned}
& P_{n}(t)=\frac{1}{2^{n} n !} \frac{d^{n}\left(t^{2}-1\right)^{n}}{d t^{n}}, \quad n=1,2, \cdots \\
& P_{0}(t)=1
\end{aligned}
$$

In the interval $[-1,1]$, the Legendre polynomial can be obtained by the following recurrence relations :

$$
P_{n+1}(t)=\frac{2 n+1}{n+1} t P_{n}(t)-\frac{n}{n+1} P_{n-1}(t) \quad n=1,2, \cdots
$$

$$
P_{0}(t)=1 \quad P_{1}(t)=t
$$

By transformation, the Legendre polynomial can be obtained in the interval $[a, b]$ :

$$
t=\frac{2 x-(a+b)}{b-a}, \quad x \in[a, b], t \in[-1,1]
$$

$$
\tilde{P}_{n}(x)=P_{n}(t)=P_{n}\left(\frac{2 x-(a+b)}{b-a}\right)
$$

Giving a group of data, $\left\{\left(x_{i}, y_{i}\right)\right\}_{i=1, \text { an }}^{m} a=x_{1}<x_{2}<\cdots<x_{m}=b$ Let $\tilde{P}_{k}(x), k=0,1, \cdots, n$ as the basis function, $c_{0}, c_{1}, \cdots, c_{n}$ is minimum value point of $\varphi(x)=c_{0}+c_{1} \widetilde{P}_{1}(x)+\cdots+c_{n} \widetilde{P}_{n}(x)$

$$
\begin{gathered}
E_{2}\left(c_{0}, c_{1}, \cdots c_{n}\right)=\sum_{i=1}^{m}\left|y_{i}-\varphi\left(x_{i}\right)\right|^{2} \\
=\sum_{i=1}^{m}\left[\sum_{j=0}^{n} c_{j} \widetilde{P}_{j}\left(x_{i}\right)-y_{i}\right]^{2}
\end{gathered}
$$

where $\tilde{P}_{j}=\left[\tilde{P}_{j}\left(x_{1}\right), \cdots \tilde{P}_{j}\left(x_{m}\right)\right]^{T}, j=0,1, \cdots, n$, $y=\left[y_{1}, \cdots y_{m}\right]^{T}, C=\left[c_{0}, \cdots, c_{n}\right]^{T}$. therefore its normal equation of the least square $G C=b$, Where $G=\left[\left(\tilde{P}_{j}, \tilde{P}_{k}\right)\right],\left(\tilde{P}_{j}, \tilde{P}_{k}\right)$ refers to the inner product of $\tilde{P}_{j}$ and $\widetilde{P}_{k} ; \quad b=\left[\left(y, \widetilde{P}_{0}\right), \cdots,\left(y, \widetilde{P}_{n}\right)\right]^{T}$. The condition number of the coefficient matrix of this normal equation is very tiny, so the fitting accuracy and practicability is high.

II. THE PREDICTION OF ELECTRICITY CONSUMPTION PER CAPITA

\section{A. Fitting Function}

The data of electricity consumption per capita from 1983 to 2013 is analyzed and electricity consumption per capita from 2014 to 2018 is predicted by the least square fitting method. Data from 1983 to 2013 is as Table 1. 
TABLE1 THE DATA OF ELECTRICITY CONSUMPTION PER CAPITA FROM 1983 TO 2013

$\mathrm{X}_{\mathrm{kwh}}$

\begin{tabular}{cc||cc||}
\hline Year & Yield & Year & Yield \\
\hline 1983 & 13.4 & 1999 & 108.6 \\
1984 & 15.3 & 2000 & 115.0 \\
1985 & 21.2 & 2001 & 126.5 \\
1986 & 23.2 & 2002 & 138.3 \\
1987 & 26.4 & 2003 & 159.7 \\
1988 & 31.2 & 2004 & 184.0 \\
1989 & 35.3 & 2005 & 221.3 \\
1990 & 42.2 & 2006 & 255.6 \\
1991 & 47.2 & 2007 & 308.3 \\
1992 & 54.9 & 2008 & 331.9 \\
1993 & 62.5 & 2009 & 336.0 \\
1994 & 72.7 & 2010 & 383.1 \\
1995 & 83.5 & 2011 & 418.1 \\
1996 & 87.7 & 2012 & 460.4 \\
1997 & 98.6 & 2013 & 515.0 \\
1998 & 104.2 & & \\
\hline
\end{tabular}

By using the Matlab mathematic software, the fitting result of $\mathrm{GM}(1,1)$ as follows:

$$
p(x)=200.7845 \exp (0.10931 \mathrm{x})-187.3845
$$

The fitting result of Cebyshev polynomial least square is that

$$
\text { Let } \quad a=1983 \quad b=2013
$$$$
t=\frac{2 x-(a+b)}{b-a} \text {, then }
$$

$$
\begin{aligned}
\phi(t)= & 153.8331+218.8283 t \\
& +109.0378 \times\left(3 t^{2}-1\right) / 2 \\
& +34.9373\left(5 t^{3}-3 t\right) / 2
\end{aligned}
$$

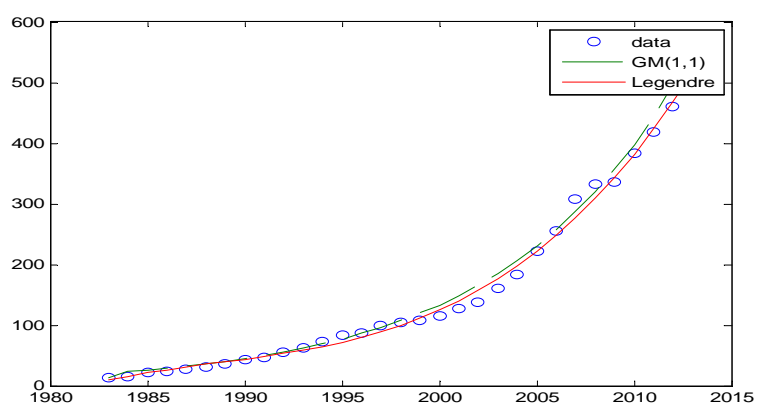

Fig.1 The actual fitting curve energy consumption per capita From 1983 to 2013
It can be seen from Fig. 1 that the effect of $\operatorname{GM}(1,1)$ is bad, but the fitting result of the Cebyshev polynomial least square is more accurate.

\section{B. Evaluation on the Fitting Results}

1) Evaluation on unbiased variance ${ }^{[5]}$.

The formula is used to evaluate the unbiased variance is:

$$
M S E=\frac{1}{N} \sum_{i=1}^{N} e_{i}^{2}=\frac{1}{N} \sum_{i=1}^{N}\left(y_{i}-\hat{y}_{i}\right)^{2}
$$

Where $\hat{y}_{i}$ is the fitting evaluation value of ${ }^{x_{i}}$. When using the unbiased variance to evaluate, the usually followed principle is the smaller the mean square error, the better the method used. Of $\mathrm{GM}(1$, 1) , $M S E$ MSE $=569.8124$, but the linear least square of the Legendre polynomial , $M S E=315.8785$.

2) Determination of the coefficient

The formula of the determination coefficient is as follows:

$$
R^{2}=1-\frac{\sum\left(y_{i}-\hat{y}_{i}\right)^{2}}{\sum\left(y_{i}-\bar{y}\right)^{2}}
$$

In the formula, $\bar{y}$ is the average value of $y_{i}$. When using the determination coefficient $R^{2}$ to evaluate, usually we follow the principle that $R^{2}$ closes to 1 is the best method. $\operatorname{GM}(1,1)$ is $R^{2}=0.9879$, but the linear least square Legendre polynomial is $R^{2}=0.9948$.

\section{CONCLUSIONS AND DISCUSSIONS}

Firstly, when analyzing the data of scientific research, the linear least square of the Legendre polynomial is better method.

Secondly, by establishing the Legendre polynomial least square model of the data of energy consumption per capita from 1983 to 2013, electricity consumption in annual per capita from 2014 to 2018 can be predicted, which is 568.9249kwh, 625.1516kwh, 685.4718kwh, 750.0408kwh, $819.0140 \mathrm{kwh}$, respectively.

Thirdly, with the world energy shortage, but also with low carbon and improvement of consciousness environmental protection and energy conservation, people naturally explore to seek new energy, especially renewable energy. In recent years, photovoltaic power generation is a kind of energy, which can provide power to the people's life and economic growth. Photovoltaic power generation has gradually been associated with industry, agriculture, service industry project and people's life 


\section{REFERENCES}

[1] L.Fu, gray system theory and its application [M], Science and Technology Literature Press, 1991

[2] J.L. Deng, the basic method of gray system [M], Huazhong University Press, 1992: P131-135.

[3] C. S. LIN. Numerical Analysis [M]. Beijing:Science Press .2007:P174-178.

[4] M. T. HEATH. Scientific Computing [M].Beijing: Tsinghua University Press .2005:P59-61

[5] S. MAO CHENG YM.Tutorial of Probability and mathematical statistics [M]. Beijing: Higher Education Press .2004:418-419.

[6] C. F. DING. Application of Combination forecasting method in China's grain output prediction [J]. Research of Agricultural Modernization, 2007 (3):10-103. 